\title{
Morte por AIDS ou morte materna: a classificação da mortalidade como prática social
}

\author{
Death by AIDS or maternal death: \\ classification of mortality as a social practice
}

Rosa Dalva F. Bonciani 1

Mary Jane P. Spink 2

\footnotetext{
1 Coletivo Feminista Sexualidade Saúde. Rua Bartolomeu Zunega 44, São Paulo, $S P$ 05426-020, Brasil. boncianir@uol.com.br 2 Pontifícia Universidade Católica de São Paulo. Rua Monte Alegre 984, São Paulo, $S P$ 05014-901, Brasil. mjspink@pucsp.br
}

\begin{abstract}
This paper analyzes the decision on whether to include deaths of HIV+ pregnant women in the classification of maternal mortality. The study focuses on deaths of childbearingage women in São Paulo in 1998, investigated by the State's Central Committee on Maternal Mortality (CCMM). Working from a social constructionist perspective, the research was based on documental analysis and interviews with the president of the CCMM and members of one regional committee. The analysis focused on the selection, investigation, and classification of maternal death, with special attention to the negotiation among various actors involved in the classificatory procedures. The data suggest that in the deaths of HIV+ women, other factors are present including the precedence of HIV status over maternal death and the moral and symbolic aspects of AIDS. The results suggest that improving data through a better understanding of decisions to include or exclude cases is only one side of the issue; it is also important to improve quality of care in pregnancy and childbirth in order to prevent maternal death.
\end{abstract}

Key words Maternal Mortality; Acquired Immunodeficiency Syndrome; HIV

Resumo O artigo trata da decisão de inclusão ou exclusão de óbitos de mulheres em idade fértil, com HIVIAIDS, nas estatísticas de mortalidade materna. Foram analisados os casos referentes ao ano de 1998, investigados pelo Comitê Central de Mortalidade Materna do Município de São Paulo (CCMM-MSP). Tendo como enquadre a abordagem construcionista, a pesquisa utilizou como fonte de dados, relatórios e entrevistas com o presidente do CCMM-MSP e membros de um comitê municipal regional. Buscou-se apontar aspectos presentes na seleção, investigação e classificação final das mortes maternas investigadas, focalizando os espaços de negociação entre os vários atores envolvidos no processo. A análise sugere que, quando se trata de óbitos de mulheres com HIVIAIDS, interferem ainda outros fatores como a precedência do status soropositivo para o HIV e os aspectos morais e simbólicos da AIDS. Concluiu-se que o aperfeiçoamento das estatísticas por meio da melhor compreensão da decisão de inclusão ou exclusão de casos é apenas um lado da questão; faz-se necessário também, melhorar a qualidade da assistência ao ciclo gravídico-puerperal para evitar a ocorrência dessas mortes.

Palavras-chave Mortalidade Materna; Síndrome de Imunodeficiência Adquirida; HIV 


\section{Introdução}

O estudo da inclusão ou exclusão da classificação da morte de mulheres em idade fértil, com HIV/AIDS nas estatísticas de mortalidade materna é uma demanda atual em nosso meio, por três fatores relacionados entre si. O primeiro é o número elevado de mulheres com HIV/ AIDS (Programa Estadual de DST/AIDS-SP, 2002). Em segundo lugar está o fato de que a maioria das mulheres infectadas encontra-se em idade reprodutiva. E, finalmente, há escassez de estudos com esse enfoque na literatura científica, uma vez que a maioria dos estudos relativos à gravidez e AIDS prioriza a problemática da transmissão materno-infantil do HIV.

$\mathrm{O}$ aprimoramento das estatísticas de morte por causa materna (causas relacionadas à gravidez, parto e puerpério) se dá por meio da investigação dos óbitos de mulheres em idade fértil por comitês de mortalidade materna. Segundo o Ministério da Saúde (MS, 1994), esses comitês têm, como objetivo, a intervenção nas decisões sobre ações médico-administrativas nos diferentes níveis de serviços que possam levar à redução das mortes maternas.

Acompanhando o processo de classificação de morte por causa materna pelo comitê, consideramos que, nos casos de mulheres com HIV/AIDS, há duas dificuldades. De um lado, temos as regras de seleção de causa de morte da Classificação Estatística Internacional de Doenças e Problemas Relacionados à SaúdeCID-10 (OMS, 1994), que dão prioridade para classificar a AIDS como causa de morte. De outro, há a força de caráter moral e simbólico em torno da AIDS, observada entre profissionais da saúde que usam o termo "aidética" para se referir a mulheres com HIV/AIDS, ou expressões como, se tem AIDS, não deveria engravidar (Bonciani, 2001).

Observamos que a orientação seguida pelos membros do comitê no processo da investigação, levava-os a classificar as mortes de mulheres com HIV/AIDS como decorrentes da AIDS, em detrimento da causa materna. Esse fato chamou nossa atenção para o risco de cristalizar-se o entendimento que, tratando-se de AIDS, não se trataria de morte por causa materna, com possíveis conseqüências para as ações de intervenção e prevenção dessas mortes. Assim, aprofundar a compreensão sobre a inclusão ou exclusão da classificação da morte de mulheres com HIV/AIDS nas estatísticas de mortalidade materna foi a questão motivadora desta pesquisa.

O presente estudo foi desenhado no enquadre da abordagem construcionista, em que o processo de classificação é entendido como uma prática social que é histórica e socialmente contextualizada. Isso implica entender as classificações como espaços de produção de sentido, assim como tomar o processo de classificação como uma complexa negociação que envolve uma variedade de atores sociais: paciente, familiares, profissionais de saúde, membros da sociedade civil organizada que atuam na área da saúde e membros dos comitês de mortalidade materna.

A análise aqui realizada buscou desfamiliarizar as práticas classificatórias, situando-as como práticas sociais produtoras de sentidos e de conhecimentos que têm conseqüências para a ação. A pesquisa sobre produção de sentidos, segundo Spink \& Medrado (1999), é um empreendimento sociohistórico e exige o esforço transdisciplinar de aproximação ao contexto cultural e social em que se inscreve um determinado fenômeno social.

O contexto da problemática da mortalidade materna, bem como da morte materna no contexto da AIDS, foram discutidos na Dissertação de Mestrado de Bonciani (2001). O objetivo deste artigo é apresentar os principais resultados da pesquisa realizada para compreender a decisão de inclusão ou exclusão de óbitos de mulheres com HIV/AIDS nas estatísticas de morte materna, por meio da análise dos casos investigados no Município de São Paulo para o ano de 1998.

\section{Metodologia}

Para compreender a decisão de inclusão ou exclusão de óbitos de mulheres com HIV/AIDS nas estatísticas de morte materna, utilizamos os dados provenientes de quatro fontes: (1) o relatório do Comitê Central de Mortalidade Materna do Município de São Paulo (CCMM-SP), para o ano de 1998, da Secretaria Municipal da Saúde de São Paulo (SMS-SP, 1999); (2) duas entrevistas com o profissional responsável pela seleção das Declarações de Óbito (DO) de 1998 que, à época da pesquisa, ocupava o cargo de Presidente do CCMM-SP; (3) os resumos da investigação e classificação de dois dos cinco óbitos de mulheres com HIV/AIDS que foram pesquisados no ano de 1998, e (4) a discussão desses dois casos com os integrantes do Comitê Regional de Mortalidade Materna.

Como primeira fonte, foi utilizado o relatório do CCMM-SP referente ao ano de 1998, porque continha os dados mais recentes à época da pesquisa e apresentava o maior número de casos com HIV/AIDS identificados como mor- 
te materna até então. Além disso, tendo participado do comitê regional há seis anos, foi neste ano que observamos uma ênfase maior no debate em torno da inclusão ou exclusão de óbitos de mulheres com HIV/AIDS nas estatísticas de morte materna. As informações contidas no relatório sobre a quantidade de DO de mulheres de 10 a 49 anos, selecionadas para investigação e, segundo o resultado encontrado pelo CCMM-SP, foram utilizadas para construir a Tabela 1.

Como segunda fonte, foram realizadas duas entrevistas com o profissional que, além de ser responsável, na época em que foi realizada a pesquisa, pela seleção das DO de mulheres em idade fértil para investigação de morte materna, participava da decisão do CCMM-SP sobre a classificação final das mortes maternas. A primeira entrevista visou a entender se havia diferença de critérios na seleção das DO de mulheres em idade fértil sem diagnóstico de HIV/ AIDS em relação às mulheres com HIV/AIDS.

A segunda entrevista buscou aprofundar a compreensão da decisão do CCMM-SP, após análise de dois óbitos de mulheres com HIV/ AIDS, de excluir um e incluir o outro na estatística de morte materna, conforme os resumos dos casos apresentados na Tabela 2. Essa tabela foi construída com base nos dados do relatório da reanálise dos casos selecionados e que constituiu nossa terceira fonte de informação.

Finalmente, de modo a entender os espaços de negociação existentes no que diz respeito à classificação de óbitos de mulheres com HIV/AIDS quanto à morte materna, realizamos uma discussão com os integrantes do Comitê Regional de Mortalidade Materna (CRMM) a respeito da reclassificação desses dois casos pelo CCMM-SP. Essa discussão constituiu nossa quarta fonte de informação.
A decisão de inclusão ou exclusão de óbitos das estatísticas de mortalidade materna, objetivo principal desta pesquisa, foi abordada a partir de três eixos: (1) a seleção dos casos para investigação, (2) os critérios para seleção e (3) a investigação da morte de mulheres com HIV/ AIDS quanto à causa materna.

\section{Aspectos éticos}

O protocolo da pesquisa foi analisado e aprovado pelo Comitê de Ética em Pesquisa da Faculdade de Saúde Pública da Universidade de São Paulo, de acordo com os requisitos da Resolução CNS/196/96.

Apoiando-se fundamentalmente em documentos de domínio público e entrevistas com pessoas que ocupavam cargos públicos, as estratégias de coleta e análise de dados buscaram assegurar a condução ética do estudo, preservando a confidencialidade e a privacidade de modo a garantir a proteção à imagem e auto-estima.

\section{Resultados}

Para analisar o processo de seleção das DO para investigação e classificação de morte materna, utilizamos as categorias definidas no Manual de Instrução (volume 2) da CID-10 (OMS, 1994):

- Morte materna: morte de uma mulher durante a gestação ou dentro de um período de 42 dias após o término da gestação, independente da duração ou da localização da gravidez, devida a qualquer causa relacionada com ou agravada pela gravidez ou por medidas em relação a ela, porém não devida a causas acidentais ou incidentais. As mortes maternas,

Tabela 1

Distribuição das mortes de mulheres de 10 a 49 anos, segundo resultado da investigação pelo Comitê de Mortalidade Materna, Município de São Paulo, Brasil, 1998.

\begin{tabular}{|c|c|c|c|}
\hline Óbitos & HIV/AIDS & Demais causas & Total \\
\hline Mortes maternas incluídas na taxa de mortalidade materna & 4 & 123 & 127 \\
\hline $\begin{array}{l}\text { Casos com gravidez, não incluídos na taxa de mortalidade } \\
\text { materna }\end{array}$ & 1 & 44 & 45 \\
\hline Negativos para gravidez & 0 & 552 & 552 \\
\hline Casos pendentes & 0 & 17 & 17 \\
\hline Total de declarações de óbito selecionadas & 5 & 736 & 741 \\
\hline
\end{tabular}

Fonte: SMS-SP (1999). 
por sua vez, podem ser subdivididas em dois grupos:

- Mortes obstétricas diretas: aquelas resultantes de complicações obstétricas na gravidez, parto e puerpério, devidas a intervenções, omissões, tratamento incorreto ou a uma cadeia de eventos resultantes de qualquer das causas acima mencionadas.

- Mortes obstétricas indiretas: aquelas resultantes de doenças existentes antes da gravidez ou de doenças que se desenvolveram durante a gravidez, não devidas a causas obstétricas diretas, mas que foram agravadas pelos efeitos fisiológicos da gravidez.

Utilizamos ainda, a categoria Morte Materna não Obstétrica (também chamada por alguns autores como "morte não relacionada"), que vem sendo utilizada para classificar as mortes durante a gravidez, parto ou puerpério, associadas a causas incidentais ou acidentais e que, conforme o Ministério da Saúde (1994), devem ser relacionadas à parte.

Casos selecionados, investigados e classificados pelo CCMM-SP em 1998

Em 1998, ocorreu no Município de São Paulo, um total de 4.347 óbitos femininos, de 10 a 49 anos, que inclui todas as causas. Desse total, 403 óbitos tinham causas relacionadas ao HIV/ AIDS, segundo o Programa de Aprimoramento das Informações de Mortalidade do Município de São Paulo (PRO-AIM), e os demais 3.944, englobaram todas as demais causas. Das 741 declarações de óbito selecionadas para investigação de morte materna, apenas 5 eram de mulheres com HIV/AIDS.

Dos 741 casos selecionados para investigação (Tabela 1), 127 foram classificados como morte materna, categoria que inclui todas as mortes obstétricas diretas e indiretas, ocorridas durante a gestação ou até 42 dias após o seu término. É com base nessas mortes que se dá o cálculo da taxa de mortalidade materna (TMM) - ou coeficiente de mortalidade materna (CMM). Quatro dessas mortes maternas eram de mulheres com HIV/AIDS. Outros 45 casos com gravidez confirmada, apresentados na Tabela 1, não entram no cálculo da taxa de mortalidade materna. Em 552 casos pesquisados, não havia gravidez envolvida por ocasião do óbito ou nos últimos doze meses que o antecederam; eles foram então considerados negativos, ou seja, sem nenhuma relação com morte materna. Havia 17 investigações ainda não concluídas até o fechamento do relatório.
Os critérios de seleção das DO de mulheres em idade fértil para a investigação de morte materna pelo CCMM-SP

A análise do processo de seleção das DO de mulheres em idade fértil para a investigação de morte materna, teve por base as informações obtidas na entrevista com o profissional responsável por essa seleção no CCMM-SP. Buscou-se, nesta etapa da análise, entender se havia diferença de critérios na seleção das DO de mulheres em idade fértil sem diagnóstico de HIV/AIDS em relação às mulheres com HIV/ AIDS.

\section{- Seleção das DO de mulheres sem diagnóstico de HIV/AIDS}

O entrevistado referiu que a seleção é feita no PRO-AIM, onde são avaliados todos os casos de morte de mulheres entre 10 e 49 anos, sendo selecionados todos os casos de morte materna declarada e todas as causas suspeitas. Como exemplo de causas suspeitas, são citadas por ele: crise convulsiva, processos infecciosos, acidente vascular cerebral (AVC), tromboembolismo pulmonar, infarto, cardiopatias, edema agudo de pulmão e hipertensão arterial. Refere tratar-se de uma lista bem ampla a ser utilizada para selecionar os casos, estratégia que, segundo seu depoimento, tem conseqüências para a prática de seleção de casos para investigação:

“Cabe aqui um parênteses, por causa do seguinte: se a gente for ..., se nós nos atermos a essa lista, ipsis literis, a quantidade de casos ... a ser pesquisada é muito grande e nós não temos capacidade operacional para investigar esses casos. Os Comitês de Morte Materna, na maioria das regionais, (....) não conseguem dar conta do volume (....). Então, pela nossa própria vivência, experiência como médico obstetra, nós procedemos a uma segunda seleção. Por exemplo, um quadro de AVC decorrente de uma hipertensão arterial numa mulher de 49 anos; $a$ chance dessa mulher estar grávida é pequena, em comparação com uma mulher de 20, 30 anos, então esse caso, geralmente, é deixado de lado (....), mesmo que ocorra perda de casos positivos para morte materna, (...) não é significativa. Então, no cômputo geral, pela própria vivência..., (....) desde 1995, o coeficiente de morte materna alterou muito pouco, mesmo ampliando esse pool de investigação".

Observa-se, assim, que a própria seleção dos casos a serem investigados está submetida a decisões tomadas com base em contingências previamente avaliadas e julgadas por quem 
a executa. Isso sugere que a prática classificatória, sendo apoiada na "própria vivência", tem caráter de construto social.

\section{- Seleção das DO de mulheres com HIV/AIDS}

Respondendo à nossa pergunta sobre como são selecionados os óbitos de mulheres com HIV/AIDS para investigação de morte materna, o entrevistado referiu que a AIDS é considerada como morte materna não relacionada por definição da OMS, a não ser que haja o envolvimento de uma causa gravídica provocando o óbito, e assim exemplificou:

"Uma paciente aidética que tem uma crise convulsiva decorrente de uma eclâmpsia. Então, o que provocou o óbito dessa paciente foi a eclâmpsia, não foi a AIDS. Então, (....) morte materna é... direta, no caso. A mesma coisa vale para cardiopatias, infecção puerperal, tudo isso; cada caso é analisado para poder ser feita a classificação. Hoje, devido ao número elevado de casos de AIDS, se não houver escrito na DO que ela estava grávida, ou não estiver explicitado no campo "causa do óbito" que ela estava grávida, nós não selecionamos o caso para investigação. Eu tenho outros casos mais importantes para serem investigados, já que a AIDS é considerada como não relacionada."

A seleção dos casos suspeitos de morte materna fundamenta-se no uso de uma lista de causas justamente devido ao sub-registro da informação, tanto da causa materna, quanto do estado gravídico nas DO. Entre as mortes maternas de 1998, esse sub-registro foi da ordem de $60 \%$, segundo a Secretaria Municipal da Saúde de São Paulo (SMS-SP,1999). Portanto, basear-se na informação sobre a existência de gravidez nas DO de mulheres com HIV/AIDS parece sugerir a operação de critérios diferenciados para a seleção dos casos.

\section{A investigação da morte de mulheres com HIV/AIDS quanto à causa materna}

Entre os cinco óbitos de mulheres com HIV/AIDS investigados pelos comitês municipais de mortalidade materna de São Paulo, em 1998, elegemos dois para análise mais aprofundada de modo a compreender a decisão final da reclassificação de inclusão de um e exclusão do outro na estatística de mortalidade materna. Os resumos da investigação fornecidos pelo CCMM-SP, são apresentados na Tabela 2:

A discussão em torno da classificação final pelo CCMM-SP dessas duas mortes - uma como obstétrica indireta e a outra como não obstétrica - foi considerada ilustrativa da complexidade do processo de decisão de inclusão ou exclusão de óbitos de mulheres com HIV/AIDS nas estatísticas de morte materna.

Dentre esses dois casos, destacamos o óbito de Araci, que foi investigado pelo CRMM do

\begin{tabular}{|c|c|c|}
\hline & Anita* & Araci* \\
\hline Idade & 30 & 31 \\
\hline \multirow[t]{5}{*}{ Atestado médico do óbito** } & Insuficiência respiratória aguda & Insuficiência respiratória \\
\hline & DPOC & Pneumonia intersticial \\
\hline & Tuberculose pulmonar & \\
\hline & HIV positivo & \\
\hline & Gestante de 28 semanas & \\
\hline \multirow[t]{3}{*}{ Achados da investigação } & História de uso de drogas & Idade gestacional $=25$ semanas \\
\hline & e tratamento incompleto/TBC & + HIV positivo + Pneumonia intersticial \\
\hline & HD: TBC + BCP + Insuficiência respiratória & + Insuficiência respiratória \\
\hline Classificação & Obstétrica indireta & Não obstétrica \\
\hline
\end{tabular}

Fonte: SMS-SP (1999)

* Nomes fictícios.

** Conforme seqüência colocada nos resumos dos casos pelo Comitê Central de Mortalidade Materna - São Paulo.

DPOC = Doença Pulmonar Obstrutiva Crônica; HD = Hipótese Diagnóstica; TBC = Tuberculose; BCP = Broncopneumonia. 
qual participamos. Portanto, acompanhamos de perto a pesquisa e discussão deste caso.

O CRMM já havia sido informado sobre a ocorrência e o local do óbito, bem como sobre a sorologia positiva para o HIV, pelo Centro Municipal de Referência de DST/AIDS onde Araci fora atendida anteriormente. Assim sendo, mesmo antes de receber a cópia da DO, o CRMM antecipou o início da investigação solicitando à Maternidade o acesso às informações. A cópia da DO chegou ao CRMM seis meses após a ocorrência do óbito. No campo das causas de morte do Atestado de Óbito (AO) estavam declaradas: Insuficiência Respiratória e Pneumonia Intersticial. O HIV não foi mencionado.

A pesquisa desse caso não fugiu à regra das dificuldades encontradas nas investigações de morte materna em geral, sobretudo no que diz respeito à obtenção das informações sobre o atendimento recebido pela mulher nos serviços, sendo estas necessárias para a compreensão da evolução do caso. Do início da investigação até a obtenção das informações sobre o pré-natal, transcorreu um ano e meio. Por meio do estudo detalhado e reconstituição histórica do caso (cf. arquivo do CRMM), foi possível acompanhar a peregrinação de Araci nos vários serviços de saúde pelos quais passou, desde o início da gravidez até sua morte.

Além dessas dificuldades, a discussão no CRMM sobre a classificação desse óbito e sua inclusão ou exclusão na categoria de morte materna suscitou um debate em decorrência do fato específico de tratar-se de uma mulher com HIV/AIDS. Entre as informações que embasavam o processo de classificação, interferiam também dois fatores: a) a tendência na literatura em afirmar que a gestação não altera o curso de progressão da infecção pelo HIV em mulheres assintomáticas (MS, 1995) e b) a prioridade para classificar como causa de morte a AIDS.

Essas proposições pareciam postular que, sendo um caso de Aids, não se tratava de morte por causa materna, tendendo a considerar a classificação desses casos como Morte Materna Não Relacionada. Do CCMM-SP procedia a orientação também nesse sentido. Entretanto, o CRMM defendia classificar o óbito de Araci como Obstétrico Indireto e, diante dessa discordância, decidimos discutir sua classificação com o CCMM-SP.

Embora um de seus membros discordasse da classificação deste caso como Morte Materna Não Relacionada, o CCMM-SP defendeu a decisão de acatar a diretriz ditada pela comunidade científica internacional. Concluiu, en- tão, pela aceitação desta diretriz e a morte de Araci foi incluída no relatório referente a 1998 entre as mortes maternas não relacionadas.

\section{Discussão: a difícil arte de classificar}

Para avançar na discussão propomos situar o processo de classificação da mortalidade - entendido como uma prática social - na confluência de dois eventos: o processo de elaboração da CID e a prática classificatória propriamente dita.

Nesses dois eventos está em jogo uma complexa negociação que envolve uma variedade de atores sociais: profissionais especialistas envolvidos com o processo de elaboração da CID, pacientes, familiares, profissionais de saúde, membros da sociedade civil organizada que atuam na área da saúde e membros do comitê.

Assim, em relação à CID, com o reconhecimento da existência de ajustes e concessões requeridos para sua elaboração (Laurenti, 1991) e do limite representado pelas condições diferentes de seu uso - que faz dela o consenso possível de ser obtido entre parceiros desiguais e em diferentes momentos (Novaes, 1987), é também com um olhar crítico que vemos a submissão às diretrizes da comunidade científica internacional, observada neste estudo. Quanto à prática classificatória propriamente dita, a postura do CCMM-SP de defesa irrestrita ao princípio da comparabilidade estabelecido pela CID parece reproduzir, no nível micro, as desigualdades que ela acarreta no nível macro.

Tendo em vista esses dois aspectos do processo classificatório, a discussão dos dados desta pesquisa foi direcionada para uma maior compreensão da decisão de inclusão ou exclusão de óbitos de mulheres com HIV/AIDS nas estatísticas de morte materna. Com esta discussão não pretendemos questionar as decisões tomadas ou reclassificar os casos. Entretanto, com base nos dados apresentados na Tabela 2, procuramos dar visibilidade e compartilhar nosso estranhamento com a classificação da morte de Anita como Obstétrica indireta - e, portanto, sua inclusão na estatística de morte materna - e a morte de Araci como Não obstétrica ou Não relacionada, excluindo-a da estatística de morte materna.

Buscando aprofundar a compreensão dessa decisão do CCMM-SP realizamos uma entrevista com o seu presidente. Paralelamente, de modo a entender os espaços de negociação existentes, no que diz respeito à classificação de óbitos de mulheres com HIV/AIDS quanto à 
morte materna, realizamos uma sessão de discussão desses dois casos com os integrantes do CRMM, responsáveis pela investigação do óbito de Araci.

Relatamos, a seguir, os argumentos apresentados nesses dois momentos, para a classificação dos dois casos que nos mostraram como parece tênue a margem que separa a classificação em uma e outra categoria. Ou seja, a classificação da causa das mortes de Anita e Araci nos pareceu paradigmática da difícil arte de classificar.

Para justificar a diferença na classificação, o argumento de nosso entrevistado no CCMMSP tomou por base a diferença das patologias. No caso de Araci, uma gestante de 25 semanas, a pneumonia intersticial foi associada a um quadro de debilidade imunológica em decorrência somente da AIDS, o que levou a classificar a morte como Não Obstétrica, ou Não Relacionada, e, portanto, uma morte por AIDS. No caso de Anita, uma gestante de 28 semanas, a limitação respiratória foi considerada como sendo decorrente de uma gestação mais avançada, piorando o quadro pulmonar já comprometido pelo antecedente de um tratamento incompleto para tuberculose. Considerou-se que a broncopneumonia estava associada à tuberculose, portanto, uma patologia pré-existente que foi agravada pela gravidez. A tuberculose foi então, a base para classificar a causa de morte como Obstétrica indireta e, portanto, uma morte materna. A AIDS foi considerada apenas uma intercorrência do caso. Conforme o entrevistado:

"O enfoque é diferente, no caso da Araci, ela tinha uma pneumonia intersticial que geralmente é provocada por vírus, e a gente sabe que o vírus, ele vai se aproveitar mais do estado de debilidade. Quer dizer, a AIDS facilitou essa pneumonia que não teve origem nenhuma a não ser a AIDS. Agora, o outro caso não, no outro, ela já tinha uma patologia de base que seria a tuberculose. Há cinco anos que ela já tinha antecedente de ter tratado mal essa tuberculose, e aí a gente joga a definição que seria uma patologia pré-existente, que foi agravada pela gravidez. Chegou no terceiro trimestre, ela começou a ter um quadro de insuficiência respiratória básica, essa insuficiência respiratória infeccionou um pulmão já prejudicado por uma tuberculose, então a gente não relacionou o fato de ela ser portadora de Aids; a Aids aí virou uma intercorrência, não uma causa, por isso que ela foi classificada como indireta".

Na discussão dos dois casos no CRMM, em que participaram quatro Ginecologistas Obstetras (GO), membros do CRMM, um deles con- siderou que uma das hipóteses para o raciocínio do CCMM seria que, no caso de Anita, a AIDS não havia sido o fator predominante; que, pelo antecedente de tuberculose, ela teria morrido em decorrência de uma pneumonia bacteriana. Quanto à Araci, sua morte teria sido decorrente de uma pneumonia intersticial causada pela infecção por Pneumocystis carinii, ou seja, uma PCP decorrente da imunodepressão por causa do HIV (GO-3).

Entretanto, esse profissional concorda que a PCP é facilitada pelo somatório de efeitos decorrentes da própria gravidez, ou seja, a baixa imunidade somada à dinâmica respiratória costal superior da grávida, que predispõem à pneumonia, conforme argumento apresentado por outro GO integrante do CRMM (GO-1).

No CRMM, onde a morte de Araci fora classificada como Obstétrica Indireta, além de argumentos condizentes com os levantados pelo CCMM-SP, a discussão comparativa com o caso de Anita trouxe argumentos para a inclusão das duas na classificação de Obstétrica Indireta. Para isso o CRMM baseou-se no entendimento de que a gravidez diminui a imunidade e piora a evolução da AIDS. Isso, não em relação às portadoras do HIV assintomáticas, mas em relação àquelas que já manifestaram sintomas da doença, como entendiam ser o caso de Araci. Um dos ginecologistas obstetras argumentou que a morte em conseqüência da associação entre AIDS e gravidez deveria ser classificada como Obstétrica Indireta, assim como é a morte decorrente da associação entre Cardiopatia e gravidez (GO-1).

Em relação à morte de Araci, um dos participantes afirmou: "nós classificamos como obstétrica indireta” (GO-4). Ao que outro participante acrescenta: "O Comitê Regional, nós classificamos certo, lá eles mudaram. E nós continuamos achando que nós estamos certos" (GO-1).

Lembrando que a visão do CCMM também não está tão errada, outras ponderações foram feitas:

"É difícil, teria que analisar profundamente" (GO-3).

"É muito complicado,...você vê, às vezes a gente traz o caso aqui e a gente mesmo fica sem saber como classificar, não é? Você investigou e você não sabe como classificar; aí quando vai para eles a coisa piora porque ... ele está chegando lá..." (GO-2).

Ainda referindo-se à Araci, a discussão prossegue com o seguinte diálogo:

"Vamos supor que ela não estivesse grávida, a evolução seria igual? ... se ela não estivesse grávida, ela morreria nesse momento? Eu acho que não" (GO-1). 
"Possivelmente, não" (GO-2).

"Pode ser que sim, mas possivelmente, não. Por isso que nós classificamos como Obstétrico Indireto" (GO-1).

Diante da suposição de que Araci e Anita não estivessem grávidas, e comparando seus quadros clínicos, três dos obstetras do CRMM estavam de acordo que Anita teria mais chance do óbito pela pneumonia em conseqüência da tuberculose do que Araci, com aquele quadro de pneumonia intersticial. Um deles, referindo-se à morte materna, concluiu: "esse argumento seria válido para classificar justamente ao contrário" (GO-1).

\section{Conclusão: morte por AIDS ou morte materna?}

Diante desses dados, o que se coloca não é uma questão de certo ou errado, ou de qual seria a verdade dos fatos, mas que esse conhecimento é construído com base em interpretações sobre as informações disponíveis que também têm, na base de sua produção, outros conhecimentos e interpretações.

\section{Referências}

BONCIANI, R. D. F., 2001. Morte por Aids ou Morte Materna: A Classificação da Mortalidade como Prática Social. Dissertação de Mestrado, São Paulo: Faculdade de Saúde Pública, Universidade de São Paulo.

LAURENTI, R., 1991. Análise da informação em saúde: 1893-1993, cem anos da Classificação Internacional de Doenças. Revista de Saúde Pública, 25:407-417.

MS (Ministério da Saúde), 1994. Manual dos Comitês de Mortalidade Materna. Brasília: Coordenação Materno Infantil, MS.

MS (Ministério da Saúde), 1995. Considerações Gerais do Binômio: HIVIAIDS e Gravidez. Brasília: Programa Nacional de DST/AIDS.

NOVAES, H. M. D., 1987. Diagnosticar e Classificar O Limite do Olhar. Tese de Doutorado, São Paulo: Faculdade de Medicina, Universidade de São Paulo.

OMS (Organização Mundial da Saúde), 1994. Classificação Estatística Internacional de Doenças e de Problemas Relacionados à Saúde. Décima Revisão. Manual de Instrução, v. 2. São Paulo: Centro Colaborador da OMS para a Classificação de Doenças em Português.
Para além da possibilidade de classificações precisas nesta ou naquela categoria - tanto no caso da Anita e da Araci como de tantas outras -, coloca-se a necessidade de se refletir sobre a possibilidade de evitar a morte de mulheres no auge de sua vida produtiva e reprodutiva.

Portanto, após as considerações sobre a difícil arte de classificar e pensando para além da classificação da Morte por AIDS ou Morte Materna, cabe aqui refletir que, quando as consideramos como eventos passíveis de serem prevenidos, muda-se o foco, passando da classificação para a assistência. Mais precisamente, para uma assistência ao ciclo gravídico-puerperal com a qualidade necessária para evitar que essas mortes ocorram.

Com esse estudo pretendeu-se chamar a atenção para o risco que a exclusão de mortes maternas entre mulheres com HIV/AIDS das estatísticas da mortalidade materna poderia ter para as ações de intervenção e prevenção dessas mortes.
PROGRAMA ESTADUAL DE DST/AIDS-SP, 2002. Boletim Epidemiológico. Ano XXI, número 1, janeiro. São Paulo: Centro de Referência e Treinamento DST/AIDS

SMS-SP (Secretaria Municipal da Saúde de São Paulo), 1999. Relatório sobre o Ano de 1998 do Comitê Central de Mortalidade Materna. São Paulo: Secretaria Municipal da Saúde, Prefeitura Municipal de São Paulo.

SPINK, M. J. P. \& MEDRADO, B., 1999. Práticas discursivas e produção de sentidos: A perspectiva da psicologia social. In: Práticas Discursivas e Produção de Sentidos no Cotidiano: Aproximações Teóricas e Metodológicas (M. J. P. Spink, org.), pp. 41-61, São Paulo: Cortez Editora.

Recebido em 9 de janeiro de 2002

Versão final reapresentada em 25 de setembro de 2002 Aprovado em 5 de fevereiro de 2003 